\title{
Elliptically Shaped Quad-Ridge Horn Antennas as Feed for a Reflector
}

\author{
O. B. Jacobs, J. W. Odendaal Senior Member, IEEE, and J. Joubert Senior Member, IEEE.
}

\begin{abstract}
Quad ridge horn antennas of various geometries are considered as possible candidates for a wideband reflector antenna feed. It is demonstrated that shaping the sidewalls of the ridge horns significantly improves the radiation characteristics of the horns. The conical quad ridge horn with an elliptically shaped sidewall shows the most promise as a wideband reflector antenna feed. The radiation patterns are rotationally symmetric and the $10 \mathrm{~dB}$ beamwidth is reasonably constant over a wide frequency range.
\end{abstract}

Index Terms-Radio astronomy, horn antenna, reflector antenna, wideband, dual polarization.

\section{INTRODUCTION}

$\mathrm{T}$ ransitions from coaxial to quad ridge waveguide are often used in radio astronomy applications and can achieve very low return loss, dual polarization and wide bandwidths [1]. These transitions are typically followed by a corrugated horn antenna to obtain rotationally symmetric radiation patterns that remain fairly constant over the frequency band of the horn antenna. These stable pattern characteristics make these horns ideal candidates as feeds for large astronomy reflector antennas. The main disadvantage is the fairly limited bandwidth (2.4:1). A direct transition from quad-ridge waveguide to free-space employing a quad ridge horn results in an antenna with a much wider VSWR bandwidth, however, the radiation patterns are not rotationally symmetric with relatively high sidelobes and beamwidths that changes as a function of frequency [2] - [5].

In this letter, various shapes of quad-ridge horn antennas are investigated as a possible candidate for a wideband reflector feed antenna. Quad-ridge horn antennas typically consist of conical or pyramidal sidewalls or are even open boundary antennas without any sidewalls. Elliptically shaped TEM horns exhibit wide frequency bandwidths with reasonably constant radiation patterns (beamwidths) [6].

The wideband pattern characteristics of quad ridge horn antennas with pyramidal, conical and diagonal structures with

Manuscript received April 18, 2011.

The authors are with the Department of Electrical Electronic and Computer Engineering, University of Pretoria, Pretoria, South Africa. (e-mail: s26249822@tuks.co.za). straight and elliptically shaped sidewalls are compared. The aim is to develop a wideband reflector feed antenna with rotationally symmetric radiation patterns and constant beamwidth

\section{ANTENNA GEOMETRIES}

The antenna geometries considered are shown in Fig. 1.

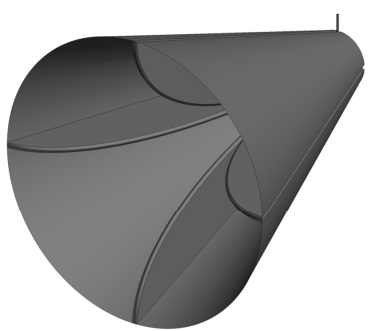

(a)

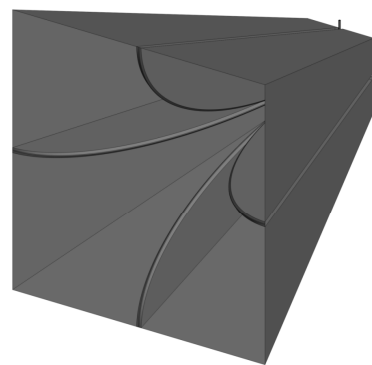

(c)

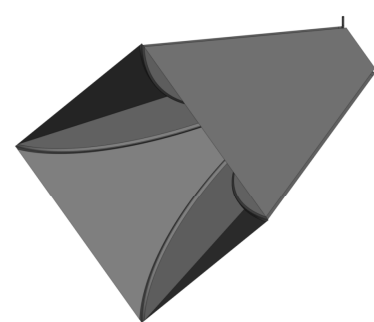

(e)

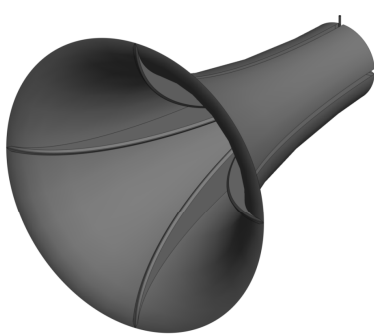

(b)

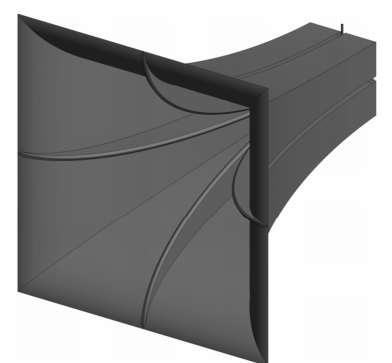

(d)

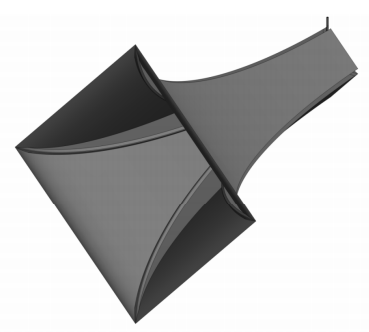

(f)
Fig. 1. Variation of the quad ridge horn antennas, a) Straight sidewall conical, b) Shaped sidewall conical, c) Straight sidewall pyramidal. d) Shaped sidewall pyramidal, e) Straight sidewall diagonal and f) Shaped sidewall diagonal.

Conical horns (without ridges) have good pattern symmetry and cross-polar performance [7]. A conical quad ridge horn 
antenna utilizing a lens to improve the radiation patterns and hence the aperture efficiency was proposed as a reflector feed in [4].

A ridge loaded pyramidal horn antenna has an aperture field that tapers along the H-plane with phase errors over both the E- and H-planes similar to a conventional horn antenna. Thus the E-plane patterns tend to have different beamwidths and higher side lobe levels than the H-plane patterns [5]. Pyramidal quad ridge horns also have large variation in beamwidth as a function of frequency.

Efforts to improve the radiation pattern of pyramidal quad ridge horn antennas included resistive inserts to reduce sidelobe levels [5] - this is not an option for radio astronomy applications where conductive losses increases system noise.

Diagonal horns (without ridges) have equal beamwidths and suppressed sidelobes as well as nearly equal E- and H-plane patterns [8]. Diagonal quad-ridge (as well as square and round) waveguides have been investigated in the past to determine characteristics such as cutoff frequencies, attenuation, impedance and field distributions [9]. Diagonal quad ridge orthomode transducers (OMT) have been investigated and provided adequate performance over standard waveguide bands [10].

The sidewalls of the conventional ridge horns above are shaped elliptically in an effort to improve on pattern symmetry and constant beamwidth behavior. The ridges are also shaped elliptically and are chamfered to obtain an impedance of $50 \Omega$.

\section{ANTENNA COMPARISON}

The antennas are modeled using FEKO [11], a commercial method of moments (MoM) solver used previously to accurately model ridge horn antennas over a wide frequency band [12].

The antenna consists of a transition from $50 \Omega$ air-filled coaxial line to ridged waveguide. This transition is identical for all the antenna types with the exception of the waveguide portion of the antenna (being either round, square or diagonal). The transition from coaxial to ridged waveguide has been optimized using FEKO for a low VSWR over at least a 4:1 frequency range.

Both the orthogonal ports are modeled, and the Sparameters of the antennas were calculated. In all cases the coupling between orthogonal ports was found to be low (less than $-40 \mathrm{~dB}$ ). The antennas were simulated from 0.5 to $4 \mathrm{GHz}$.

The effect of the different geometries and sidewalls are investigated. To ensure that only the effect of the change in sidewall profile and geometry are observed identical ridges are used for all the antennas. The waveguide dimensions and the horn apertures of the different shapes to realize identical ridges are shown in Fig. 2.

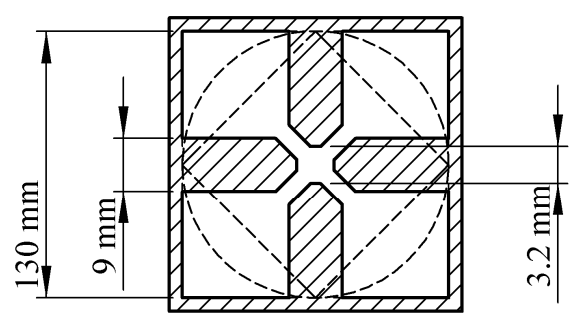

(a)

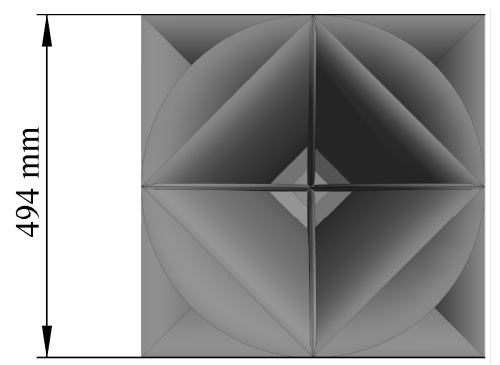

(b)

Fig. 2. (a) The ridge waveguide dimensions and (b) comparison of the apertures of the three geometries that allows for identical ridges with horn outer dimension shown.

Maintaining identical ridges results in different aperture sizes for the different horn profiles. The conical aperture area is $78.5 \%$ of the square with the diagonal area only $50 \%$ of the square. The lower operating frequencies of the antennas are also different due to the different waveguide geometries. As shown in Fig. 3 both ports of the antennas are well matched over a large portion of the frequency band.

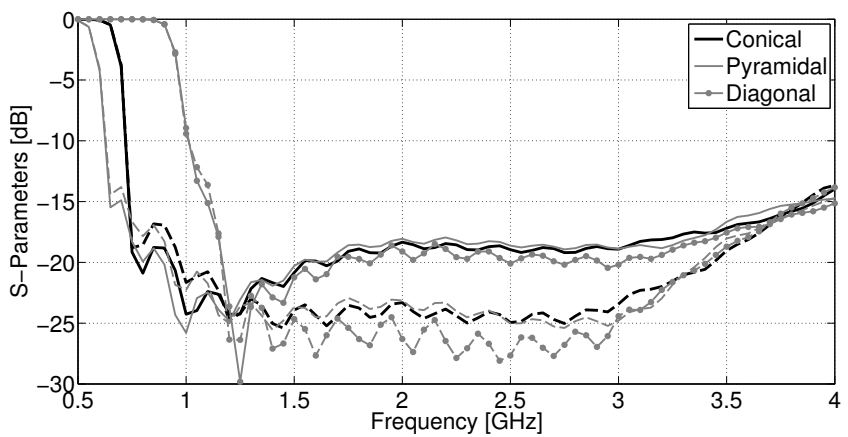

Fig. 3. S-parameters of the antennas (- Port 1 - - Port 2)

\section{COMPARISON OF RADIATION CHARACTERISTICS}

The suitability of the antennas as a wideband feed for a reflector antenna is based on the $-10 \mathrm{~dB}$ beamwidth and the difference between principal plane patterns (a measure of the rotational symmetry of the antenna). The beamwidths for the different antennas are shown in Fig. $4-6$ and summarized in Table 1. The mean and standard deviation in Table 1 is determined from 1 to $3 \mathrm{GHz}$. 


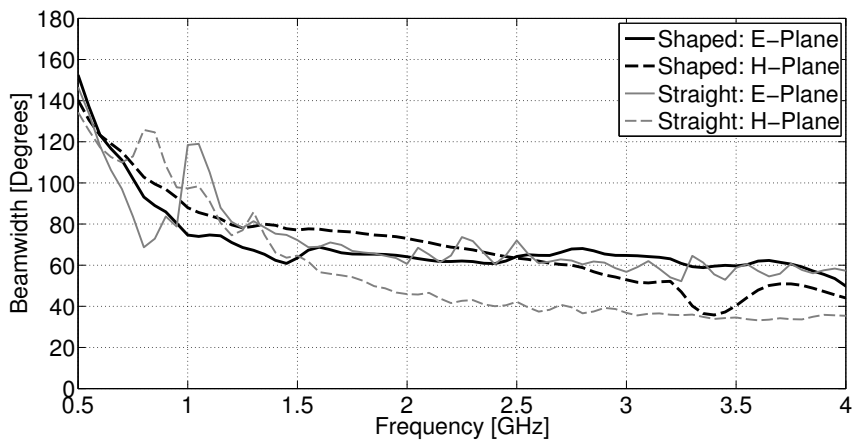

Fig. 4. $10 \mathrm{~dB}$ Beamwidth of the conical quad ridge horn antennas.

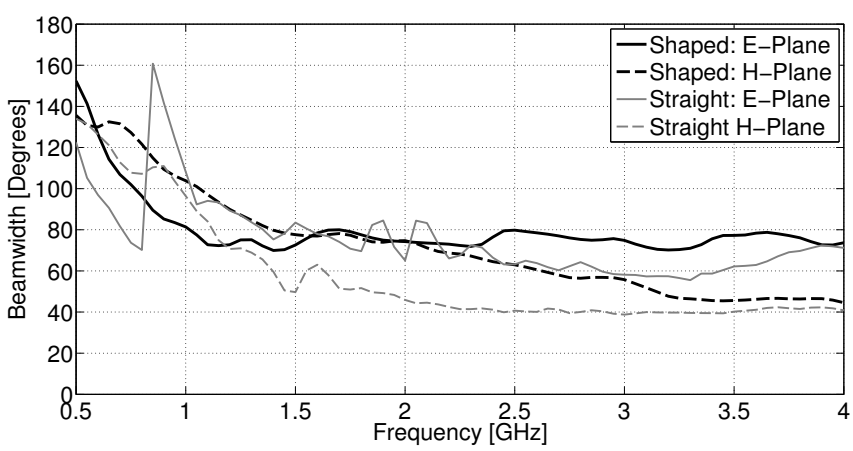

Fig. 5. $10 \mathrm{~dB}$ Beamwidth of the pyramidal quad ridge horn antennas.

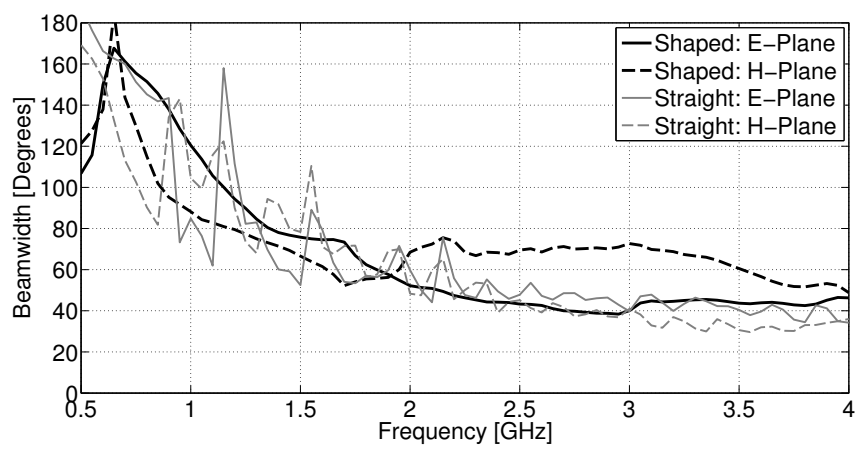

Fig. 6. $10 \mathrm{~dB}$ Beamwidth of the diagonal quad ridge horn antennas.

Sudden changes in the beamwidths indicate the presence of sidelobes close to the $10 \mathrm{~dB}$ level. This is especially undesirable as these sidelobes are close to the reflector edge illumination and could increase spillover. In Fig. $4-6$ the 10 $\mathrm{dB}$ beamwidth varies smoothly for shaped antennas indicating an improvement over the antennas with straight sidewalls.

The variation in beamwidth as a function of frequency is smaller for the pyramidal and conical antennas compared to the diagonal antennas. The differences between the E- and $\mathrm{H}$ plane beamwidths are smaller for the shaped sidewalls than for the conventional straight sidewalls. This is evident from the mean and standard deviation of the $10 \mathrm{~dB}$ beamwidth as shown in Table 1. The shaped sidewalls also improved the rotational symmetry of the radiation patterns for the conical and pyramidal horns.
The diagonal quad ridge horn antenna with straight sidewalls shows equal E- and $\mathrm{H}$-plane radiation patterns for a large portion of the bandwidth. The shaping in fact reduces the rotational symmetric performance of this horn. A frequency scaled version of the diagonal horn matched from $0.75 \mathrm{GHz}$ had a boresight gain with large variations at higher frequencies, with some pattern break up evident due to higher order modes. There was a larger difference between the principal plane mean $10 \mathrm{~dB}$ beamwidths. All dimensions except the ridge gap and ridge width were scaled by the ratio between the current and desired lower operating frequency.

The conical shaped quad ridge horn antenna has the greatest potential as a reflector antenna feed due to smaller beamwidth variation and reasonable pattern symmetry over a wide frequency band. The principal plane radiation patterns of the shaped conical quad ridge horn antenna are shown in Fig. 7.

TABLE I

COMPARISON OF MEAN AND STANDARD DEVIATION OF ANTENNA $10 \mathrm{~dB}$ BEAMWIDTH (DEGREES)

\begin{tabular}{lllll}
\hline \hline & $\begin{array}{c}\text { E-plane } \\
\text { mean }\end{array}$ & $\begin{array}{c}\text { H-plane } \\
\text { mean }\end{array}$ & $\begin{array}{c}\text { E-plane } \\
\text { standard } \\
\text { deviation }\end{array}$ & $\begin{array}{c}\text { H-plane } \\
\text { standard } \\
\text { deviation }\end{array}$ \\
\hline $\begin{array}{l}\text { Conical } \\
\text { Straight }\end{array}$ & 71.2 & 60.5 & 14.1 & 25.5 \\
$\begin{array}{l}\text { Conical } \\
\text { Shaped }\end{array}$ & 65.8 & 70.9 & 3.7 & 9.1 \\
$\begin{array}{l}\text { Square } \\
\text { Straight }\end{array}$ & 74.3 & 52.0 & 11.7 & 14.8 \\
$\begin{array}{l}\text { Square } \\
\text { Shaped }\end{array}$ & 74.8 & 66.8 & 2.7 & 12.7 \\
$\begin{array}{l}\text { Diagonal } \\
\text { Straight }\end{array}$ & 61.6 & 63.7 & 21.9 & 24.0 \\
Diagonal & 61.9 & 69.3 & 22.8 & 8.1 \\
Shaped & & & & \\
\hline \hline
\end{tabular}

\section{CONCLUSION}

Various shapes of quad-ridge horn antennas were investigated as a possible candidate for a wideband reflector feed antenna. Conventional quad-ridge horn antennas with conical, pyramidal and diagonal sidewalls were considered. It was demonstrated that shaping the sidewall significantly improves the radiation characteristics of the conical and pyramidal quad ridge horns. The conical quad ridge horn with elliptically shaped sidewalls shows the most promise as a possible candidate for a wideband reflector antenna feed. The radiation patterns are rotationally symmetric and the $10 \mathrm{~dB}$ beamwidth reasonably constant over a wide frequency range. 


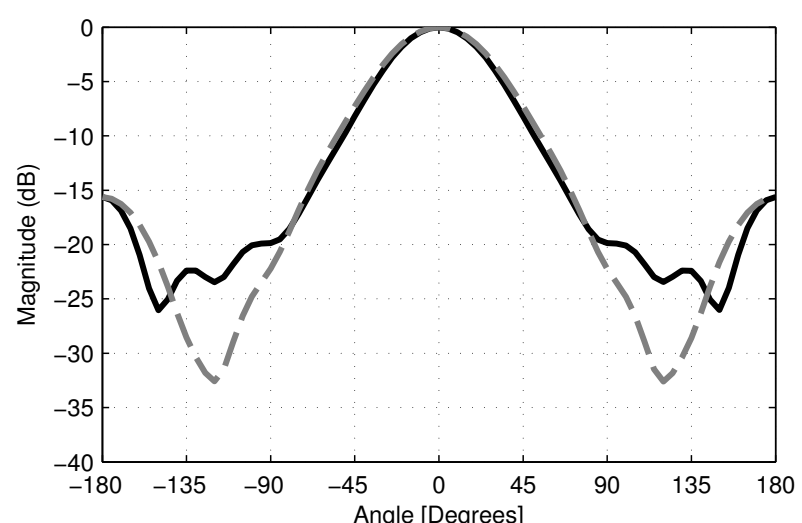

(a)

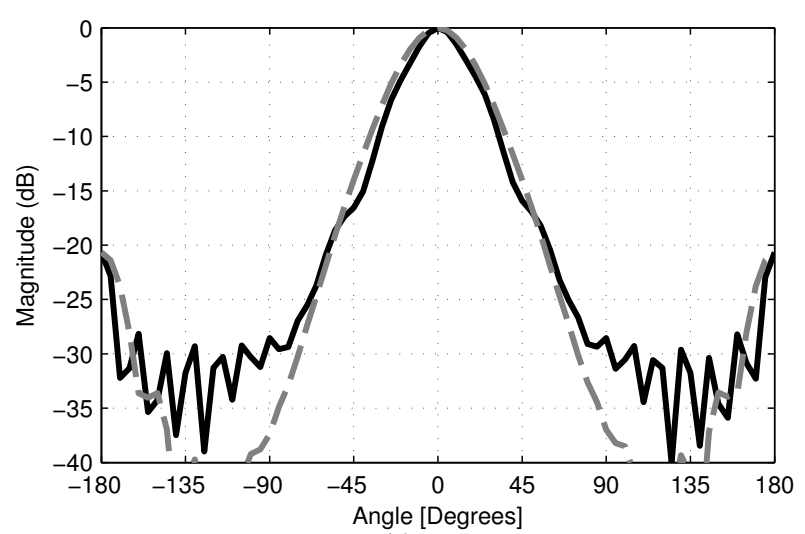

(c)

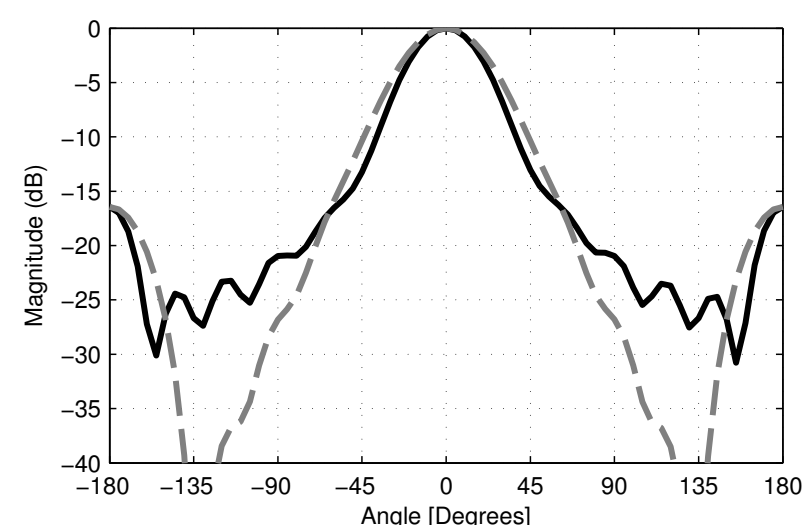

(b)

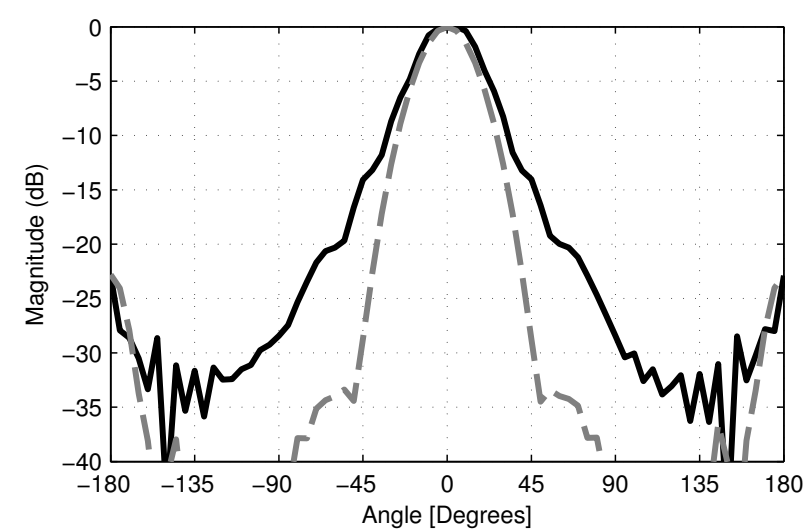

(d)

Fig. 7. The radiation patterns of the conical shaped quad ridge horn antenna (- E-Plane - - - H-Plane) at (a) $0.75 \mathrm{GHz}$, (b) $1 \mathrm{GHz}$, (c) $2 \mathrm{GHz}$ and (d) $3 \mathrm{GHz}$.

\section{ACKNOWLEDGMENT}

The authors thank EMSS-SA who provided valuable support and insight on the use of FEKO. The research was also supported by the National Research Foundation (NRF) of South Africa.

\section{REFERENCES}

[1] S.J. Skinner and G.L. James, "Wide-band orthomode transducers", IEEE Trans. Microw. Theory Tech., vol. 39, no. 2, Feb. 1991.

[2] V. Rodriguez, "A multi-octave, open-boundary, quad-ridge horn antenna for use in the S- to Ku-bands", Microwave Journal, vol. 49, no. 3, Mar. 2006.

[3] Z. Shen and C. Feng, "A new dual-polarized broadband horn antenna," IEEE Antennas Wireless Propag. Lett., vol. 4, pp. 270-273, 2005.

[4] R.J. Bauerle, R. Schrimpf, E. Gyorko, and J. Henderson, "The use of a dielectric lens to improve the efficiency of a dual-polarized quad-ridge horn from 5 to $15 \mathrm{GHz}$, IEEE Trans. Antennas Propag., vol. 57, no. 6, pp. 1822-1825 June 2009.

[5] M. Gilbert, K. Higgins, and L. Romero, "Quad-ridge Horn Utilizing Resistive Films to Reduce Sidelobes", IEEE Antennas and Propagation Society Int. Symp., 2007. pp.5684-5687, 9-15 June 2007.
[6] J.A.G Malherbe, "Frequency independent performance of elliptic profile TEM horns", Microwave and Optical Technology Letters, vol. 51, no. 3, Mar. 2009.

[7] T. S. Bird, "A Multibeam feed for the Parkes radio-telescope", IEEE Antennas Propagation Symp., pp. 966-969, Jun. 1994.

[8] C.A. Balanis, Antenna theory analysis and design, $3^{\text {rd }}$ ed., Hoboken, NJ: Wiley, 2005, pp. 794 - 796

[9] W. Sun and C.A. Balanis, "Analysis and design of quadruple-ridged waveguides", IEEE Trans. Microw. Theory Tech., vol. 42, no. 12, pp. 2201-2207, Dec. 1994.

[10] G.M. Coutts, "Wideband diagonal quadruple-ridge orthomode transducer for circular polarization detection", IEEE Trans. Antennas Propag., to be published.

[11] FEKO User's Manual Suite 6.0, EM Software \& Systems - S.A. (Pty) Ltd, Stellenbosch, South Africa, Sept. 2010.

[12] B. Jacobs, J.W. Odendaal and J. Joubert, "The effect of manufacturing and assembly tolerances on the performance of double-ridged horn antennas", J. of Electromagn Waves and Appl., vol. 24, pp. 1279 $1290,2010$. 